\title{
LIOUVILLIAN INTEGRABILITY OF GRAVITATING STATIC ISOTHERMAL FLUID SPHERES
}

\author{
ROBERTO IACONO ${ }^{2}$ AND JAUME LLIBRE ${ }^{2}$
}

\begin{abstract}
We examine the integrability properties of the Einstein field equations for static, spherically symmetric fluid spheres, complemented with an isothermal equation of state, $\rho=n p$. In this case, Einstein's equations can be reduced to a nonlinear, authonomous second order ODE for $m / R(m$ is the mass inside the radius $R$ ) that has been solved analytically only for $n=-1$ and $n=-3$, yielding the cosmological solutions by De Sitter and Einstein, respectively, and for $n=-5$, case for which the solution can be derived from the De Sitter's one using a symmetry of Einstein's equations. The solutions for these three cases are of Liouvillian type, since they can be expressed in terms of elementary functions.

Here we address the question of whether Liouvillian solutions can be obtained for other values of $n$. To do so, we transform the second order equation into an equivalent authonomous Lotka-Volterra quadratic polynomial differential system in $\mathbb{R}^{2}$, and characterize the Liouvillian integrability of this system using Darboux theory. We find that the Lotka-Volterra system possesses Liouvillian first integrals for $n=-1,-3,-5$, which descend from the existence of invariant algebraic curves of degree one, and for $n=-6$, a new solvable case, associated to an invariant algebraic curve of higher degree (second). For any other value of $n$, eventual first integrals of the Lotka-Volterra system, and consequently of the second order ODE for the mass function must be non Liouvillian. This makes quite unlikely the existence of other solutions of the isothermal fluid sphere problem with a Liouvillian metric.
\end{abstract}

\section{Introduction AND STATEMENT OF THE MAIN RESUlt}

Exact solutions of Einstein's field equations describing static, spherically symmetric fluid spheres have been sought for decades, both in cosmological contexts and as possible models of the internal structure of neutron stars. The inherent mathematical underdetermination of the problem (three equations for the four variables: density, pressure, and two metric coefficients) has allowed for the derivation of a considerable number of such solutions, even though very few of them satisfy all the requirements for physical acceptability (see, e.g., the classical work by Delgaty and Lake [1], where the 127 non cosmological solutions available at the time were classified and tested for physical relevance).

The underdetermination can be removed by specifying an additional physical constraint, such as, for example, an equation of state, but this usually makes it very

1991 Mathematics Subject Classification. Primary 34C05, 34A34, 34C14.

Key words and phrases. Liouvillian integrability, Lotka-Volterra system, gravitating static isothermal fluid spheres. 
difficult to derive explicit analytic solutions. The case of a simple isothermal equation of state,

$$
\rho=n p,
$$

has been considered since the first years of life of general relativity, leading to the discovery of two cosmological solutions: one by De Sitter, for $n=-1$, and the other, for $n=-3$, by Einstein himself (see [1]). A solution that is singular at the origin was then found by Tolman [2], and rediscovered by other authors at later times. Much more recently, Ivanov [3] has shown that the De Sitter solution can be used to solve the $n=-5$ case, exploiting a symmetry of the Einstein equations originally pointed out by Buchdhal [4]. On the other hand, no regular analytic solution with positive $n$ has ever been found.

It has been argued in [3] that this scarsity of solutions can be understood by noticing that the isothermal problem can be reduced to the solution of a second order, nonlinear, autonomous ODE that can in its turn be transformed into an Abel equation of the second kind. Then, one finds that the cases $n=-1,-3$ are precisely those for which the resulting Abel equations are known to be solvable. We note, however, that while this is certainly of help in focusing on the mathematical difficulty of the problem, it does not exclude the existence of other solvable cases, and even of other solvable cases with solutions expressible in terms of elementary functions, because a complete classification of the solvable Abel equations is still lacking.

To make further progress, here we follow a different approach. Our basic observation is that the problem at hand, for $n \neq 0$, can be reduced (see the derivation in section 2) to the Lotka-Volterra quadratic polynomial differential system

$$
\begin{aligned}
& \dot{u}=u\left(\frac{1-n}{2 n} u+\frac{n+3}{4} v+\frac{n+7}{2}\right)=P(u, v), \\
& \dot{v}=v\left(-u+\frac{1}{2} v+1\right)=Q(u, v),
\end{aligned}
$$

whose Liouvillian integrability can be studied using established results from the Darboux theory of integrability (see, e.g., [6]). We recall that, roughly speaking, Liouvillian integrable polynomial differential systems are polynomial differential systems having first integrals given by elementary functions or by integrals of elementary functions. Here a function is elementary if it is the composition of exponential functions, trigonometric functions, logarithmic functions and polynomial functions. For a rigorous definition of Liouvillian integrability see section 2 of Singer [5].

Our main result is the following one where we characterize the differential systems (2) which are Liouvillian integrable.

Theorem 1. The Lotka-Volterra quadratic polynomial differential system is Liouvillian integrable if and only if $n \in\{-1,-3,-5,-6\}$, and a Liouvillian first integral 
is

$$
\begin{array}{ll}
\frac{u(2 u-3 v-6)^{2}}{v^{3}} & \text { if } n=-1, \\
\frac{u(2 u-3 v-6)^{2}}{v^{2}} & \text { if } n=-3, \\
\frac{u\left(3(2 u-5 v)^{2}-20 u+150 v\right)^{2}}{v^{3}} & \text { if } n=-5, \\
H_{1}(u, v)-H_{2}(u, v)+H_{3}(u, v) & \text { if } n=-6,
\end{array}
$$

where

$$
\begin{aligned}
& H_{1}=\frac{6 \sqrt{|v|}\left(5 u^{4}+150 u^{2} v-45 u^{3} v+864 v^{2}-450 u v^{2}+135 u^{2} v^{2}-135 u v^{3}\right)}{u\left(u^{2}-6 u v+9 v^{2}+18 v\right)^{2}}, \\
& H_{2}=\frac{5(2 u+\sqrt{9-6 u}-3)}{\sqrt{(3-2 u)(3-u-\sqrt{9-6 u})}} \operatorname{arctanh} \sqrt{\frac{3|v|}{3-u-\sqrt{9-6 u}}}, \\
& H_{3}=\frac{5(2 u-\sqrt{9-6 u}-3)}{\sqrt{(3-2 u)(3-u+\sqrt{9-6 u})}} \operatorname{arctanh} \sqrt{\frac{3|v|}{3-u+\sqrt{9-6 u}}} .
\end{aligned}
$$

The proof of the "if" part of Theorem 1 can be found in section 4 , and of the "only if" in section 5 .

Thus, we find a new case of integrability, for $n=-6$, together with the cases with $n=-5,-3,-1$ that were already known. These four cases of integrability are of Liouvillian type, and Theorem 1 shows that there are no other cases of Liouvillian integrability for system (2). It may be worth noting that our method naturally yields the case $n=-5$, which could not be found in [3] through the approach based on the reduction of the problem to an Abel equation, since the resulting Abel equation was not in the list of the known solvable ones. Note also that for $n=-5,-3,-1$ the first integral is given by a rational function, and that this is not the case for $n=-6$.

\section{Derivation of the Lotka-Volterra system (2)}

In a spherically symmetric configuration, the Einstein equations can be reduced to the so-called Tolman-Oppenheimer-Volkoff (TOV) equation,

$$
\frac{d p}{d R}=-\frac{(\rho+p)\left(2 m+p R^{3}\right)}{2 R(R-2 m)}
$$

which relates the pressure $p$ to the density $\rho$. Here $R=R(r)$ is a generic radial coordinate, and the mass $m(R)$ is given by

$$
m(R)=\frac{1}{2} \int_{0}^{R} \rho R^{2} d R
$$

(we refer to [3] for further details, such as those concerning the normalizations). If we now specialize to the equation of state (1), it is clear that the TOV equation can be seen as a nonlinear second order ODE for $m(R)$, or some related variable. 
Following [3], we can introduce the new variables $M=m / R$ and $D=\rho R^{2} / 2$, and a new radial coordinate $\tau=\ln R$, and rewrite (3) and (4) as the autonomous system

$$
\begin{aligned}
& (2 M-1) \dot{D}=D\left[(n+5) M-2+\frac{n+1}{n} D\right], \\
& \dot{M}=-M+D
\end{aligned}
$$

where the overdot denotes derivation with respect to $\tau$. At this point, one may proceed as in [3], and reduce system (5) to a second order autonomous equation for the variable $x=M-1 / 2$. We instead rewrite (5) as

$$
\begin{aligned}
& \dot{D}=D\left(\frac{n+5}{2}+\frac{n+1}{2 n} \frac{D}{x}+\frac{n+1}{4 x}\right), \\
& \dot{x}=-x+D-1 / 2,
\end{aligned}
$$

and note that this implies

$$
\frac{d}{d \tau}\left(\frac{D}{x}\right)=\frac{n+7}{2} \frac{D}{x}+\frac{1-n}{2 n} \frac{D^{2}}{x^{2}}+\frac{n+3}{4} \frac{D}{x^{2}} .
$$

Then, we only need to introduce the new variables

$$
u=D / x=(\dot{x}+x+1 / 2) / x, \quad v=1 / x,
$$

to rewrite (7) and the second of (6) as the Lotka-Volterra system (2) of the previous section.

To complete the picture, we recall the relations that yield the two metric coefficients (see [3]) in terms of $\rho$ and $p$ :

$$
\frac{d \nu}{d R}=-\frac{1}{\rho+p} \frac{d p}{d R}
$$

and

$$
e^{-\lambda} R^{\prime 2}=\frac{1}{2}-\frac{m}{R}=-\frac{1}{v}
$$

where the prime denotes derivation with respect to the radius $r$ (the $R^{\prime}$ term would equal unity for the choice $R=r$, which yields the so-called curvature coordinates). Note that (3), (4), (9), and (10) imply that if $m$ is Liouvillian, then the metric is also Liouvillian.

We can now state more clearly the consequences of Theorem 1 on the integrability of the isothermal Einstein equations. It follows from (8), and from the construction of (6) that any first integral of (2) yields a first integral of the second order authonomous equation for $x$ derived in [3], and viceversa. Thus, when $n \neq-1,-3,-5,-6$, the second order equation for $x$ can only have non Liouvillian first integrals. This does not explicitly forbids the existence of Liouvillian solutions, but makes it quite unlikely. 


\section{Basic Results From the Darboux theory of INTEGRABility}

In this section we recall the basic definitions and results from the Darboux theory of integrability that we shall need for proving Theorem 1. For more details on this theory of integrability than the ones described in this section see the Chapter 8 of [6].

We consider polynomial differential systems in $\mathbb{R}^{2}$ defined by

$$
\dot{x}=P(x, y), \quad \dot{y}=Q(x, y),
$$

where $P$ and $Q$ are polynomials with real coefficients. We say that this polynomial differential system has degree $d$ if $d$ is the maximum degree of $P$ and $Q$. The dot denotes derivative with respect to the independent variable. Associated to the polynomial differential system (11) we have the polynomial vector field $\mathcal{X}$ given by

$$
\mathcal{X}=P(x, y) \frac{\partial}{\partial x}+Q(x, y) \frac{\partial}{\partial y} .
$$

As usual $\mathbb{C}[x, y]$ denotes the ring of all complex polynomials in the variables $x$ and $y$. Let $f=f(x, y) \in \mathbb{C}[x, y]$. We say that $f=0$ is an invariant algebraic curve of the polynomial vector field $\mathcal{X}$ if $f$ satisfies

$$
\mathcal{X} f=P \frac{\partial f}{\partial x}+Q \frac{\partial f}{\partial y}=K f
$$

for some polynomial $K=K(x, y) \in \mathbb{C}[x, y]$, called the cofactor of $f=0$. Note that the polynomial $K$ has degree at most $d-1$. Moreover if $f \notin \mathbb{R}[x, y]$ then the conjugate of $f$ provides also an invariant algebraic curve with cofactor the conjugate of $K$. Independently of working with real polynomial vector fields $\mathcal{X}$ when we study their Liouvillian first integrability we need to consider the complex invariant algebraic curves, because sometimes these complex invariant algebraic curves force the real integrability.

We say that an invariant algebraic curve $f=0$ is irreducible if the polynomial $f$ is irreducible in $\mathbb{C}[x, y]$.

Proposition 2. Suppose $f \in \mathbb{C}[x, y]$ and let $f=f_{1}^{n_{1}} \ldots f_{r}^{n_{r}}$ be its factorization into irreducible factors over $\mathbb{C}[x, y]$. Then for a polynomial differential system (11) $f=0$ is an invariant algebraic curve with cofactor $K_{f}$ if and only if $f_{i}=0$ is an invariant algebraic curve for each $i=1, \ldots, r$ with cofactor $K_{f_{i}}$. Moreover $K_{f}=$ $n_{1} K_{f_{1}}+\ldots+n_{r} K_{f_{r}}$.

An exponential factor $F$ of the polynomial vector field $\mathcal{X}$ of degree $m$ is an exponential function of the form $F=\exp (h / g) \notin \mathbb{C}$ with $g, h \in \mathbb{C}[x, y]$ satisfying

$$
\mathcal{X} F=P \frac{\partial F}{\partial x}+Q \frac{\partial F}{\partial y}=L F,
$$

for some polynomial $L$ of degree at most $d-1$, called the cofactor of the exponential factor $F$. Exponential factors of the form $\exp (h / g)$ with $g \neq 1$ appear when the multiplicity of the invariant algebraic curve $g=0$ is larger than one, and with $g=1$ 
appear when the multiplicity of the invariant straight line at infinity is larger than one, see for more details [7].

Let $U$ be an open and dense set in $\mathbb{R}^{2}$. We say that a non-constant $C^{1}$ function $H: U \rightarrow \mathbb{R}$ is a first integral of the polynomial vector field $\mathcal{X}$ on $U$, if $H(x(t), y(t))$ is constant for all values of $t$ for which the solution $(x(t), y(t))$ of $\mathcal{X}$ is defined on $U$. Clearly $H$ is a first integral of $\mathcal{X}$ on $U$ if and only if $\mathcal{X} H=0$ on $U$.

A non-constant function $R: U \rightarrow \mathbb{R}$ is an integrating factor for the polynomial vector field $\mathcal{X}$, if one of the following three equivalent conditions holds

$$
\frac{\partial(R P)}{\partial x}=-\frac{\partial(R Q)}{\partial y}, \quad \operatorname{div}(R P, R Q)=0, \quad \mathcal{X} R=-R \operatorname{div}(P, Q)
$$

on $U$. As usual the divergence of the vector field $\mathcal{X}$ is defined by

$$
\operatorname{div}(P, Q)=\frac{\partial P}{\partial x}+\frac{\partial Q}{\partial y}
$$

Knowing an integrating factor $R$ of the differential system (11) we can compute a first integral $H$ of $\mathcal{X}$ as follows

$$
H=-\int R(x, y) P(x, y) d y+h(x),
$$

where the function $h(x)$ is determined from the equality $\frac{\partial H}{\partial x}=R(x, y) Q(x, y)$.

Let $f_{i}, g_{j}, h_{j} \in \mathbb{C}[x, y]$ for $i=1, \ldots, p$ and $j=1, \ldots, q$. Then the (multi-valued) function

$$
f_{1}^{\lambda_{1}} \cdots f_{p}^{\lambda_{p}} e^{\mu_{1} g_{1} / h_{1}} \cdots e^{\mu_{q} g_{q} / h_{q}}
$$

with $\lambda_{i}, \mu_{j} \in \mathbb{C}$ is called a (generalized) Darboux function.

We say that a polynomial differential system (11) is Liouvillian integrable if it has a first integral or an integrating factor given by a generalized Darboux function, for more details see Singer [5].

Now we recall the characterization under suitable assumptions of the multiplicity of an invariant algebraic curve using the number of exponential factors associated to that curve.

Let $m$ be a positive integer. We say that an invariant algebraic curve $f=0$ of degree $k$ of a vector field $\mathcal{X}$ has (algebraic) multiplicity $m$ if $f^{m}$ is a factor of the so-called extactic polynomial of $\mathcal{X}$ but $f^{m+1}$ is not a factor of the extactic polynomial, where the extactic polynomial of $\mathcal{X}$ is the polynomial

$$
\operatorname{det}\left(\begin{array}{cccccccc}
1 & x & y & \cdots & x^{k} & x^{k-1} y & \cdots & y^{k} \\
\mathcal{X}(1) & \mathcal{X}(x) & \mathcal{X}(y) & \cdots & \mathcal{X}\left(x^{k}\right) & \mathcal{X}\left(x^{k-1} y\right) & \cdots & \mathcal{X}\left(y^{k}\right) \\
\vdots & \vdots & \vdots & \cdots & \vdots & \vdots & \vdots & \vdots \\
\mathcal{X}^{l-1}(1) & \mathcal{X}^{l-1}(x) & \mathcal{X}^{l-1}(y) & \cdots & \mathcal{X}^{l-1}\left(x^{k}\right) & \mathcal{X}^{l-1}\left(x^{k-1} y\right) & \cdots & \mathcal{X}^{l-1}\left(y^{k}\right)
\end{array}\right),
$$

where $l$ is the number of elements in the basis $\left\{1, x, y, \ldots, x^{k}, x^{k-1} y, \ldots, y^{k}\right\}$ of all polynomials of degree at most $k$. For more details on the definition and properties of the extactic polynomial, see [7]. 
Proposition 3. Let $f=0$ be an irreducible invariant algebraic curve of degree $k$ of the polynomial vector field $\mathcal{X}$. Then $f$ has multiplicity $m$ if and only if the vector field $\mathcal{X}$ has $m-1$ exponential factors $\exp \left(g_{i} / f^{i}\right)$, where $g_{i}$ is a polynomial of degree at most $i k$ and $\left(g_{i}, f\right)=1$ for $i=1, \ldots, m-1$.

The invariant straight line at infinity has multiplicity $m$ if and only if the vector field $\mathcal{X}$ has $m-1$ exponential factors $\exp \left(g_{i}\right)$, where the polynomials $g_{i}$ are linearly independent.

In the next theorem we summarize the Darboux theory of integrability that we shall use in this paper, for a proof see Chapter 8 of [6].

Theorem 4. Suppose that a polynomial differential system (11) of degree $m$ admits $p$ irreducible invariant algebraic curves $f_{i}=0$ with cofactors $K_{i}$ for $i=1, \ldots, p$, and $q$ exponential factors $\exp \left(g_{j} / h_{j}\right)$ with cofactors $L_{j}$ for $j=1, \ldots, q$.

(i) There exist $\lambda_{i}, \mu_{j} \in \mathbb{C}$ not all zero such that $\sum_{i=1}^{p} \lambda_{i} K_{i}+\sum_{j=1}^{q} \mu_{j} L_{j}=0$, if and only if the (multi-valued) function

$$
f_{1}^{\lambda_{1}} \ldots f_{p}^{\lambda_{p}}\left(\exp \left(\frac{g_{1}}{h_{1}}\right)\right)^{\mu_{1}} \ldots\left(\exp \left(\frac{g_{q}}{h_{q}}\right)\right)^{\mu_{q}}
$$

is a first integral of system (11).

(ii) If $p+q \geq 1+m(m+1) / 2$, then there exist $\lambda_{i}, \mu_{j} \in \mathbb{C}$ not all zero such that $\sum_{i=1}^{p} \lambda_{i} K_{i}+\sum_{j=1}^{q} \mu_{j} L_{j}=0$.

(iii) There exist $\lambda_{i}, \mu_{j} \in \mathbb{C}$ not all zero such that $\sum_{i=1}^{p} \lambda_{i} K_{i}+\sum_{j=1}^{q} \mu_{j} L_{j}=-\operatorname{div}(P, Q)$, if and only if function (16) is an integrating factor of system (11).

(iv) If $p+q=m(m+1) / 2$, then function (16) is a first integral if $\sum_{i=1}^{p} \lambda_{i} K_{i}+$ $\sum_{j=1}^{q} \mu_{j} L_{j}=0$, or an integrating factor if $\sum_{i=1}^{p} \lambda_{i} K_{i}+\sum_{j=1}^{q} \mu_{j} L_{j}=-\operatorname{div}(P, Q)$, under the condition that not all $\lambda_{i}, \mu_{j} \in \mathbb{C}$ are zero.

\section{The invariant algebraic Curves of System (2)}

The main steps in the proof of Theorem 1 are given in the following lemmas. 
Lemma 5. The unique invariant algebraic curves of system (2) of degree 1 are

$$
\begin{array}{lll}
u=0 & \text { with cofactor } \frac{1-n}{2 n} u+\frac{n+3}{4} v+\frac{n+7}{2} & \text { for all } n, \\
v=0 & \text { with cofactor }-u+\frac{1}{2} v+1 & \text { for all } n, \\
2 u-3 v-6=0 & \text { with cofactor }-u+\frac{1}{2} v & \text { if } n=-1, \\
2 u-3 v-6=0 & \text { with cofactor }-\frac{2}{3} u+\frac{1}{2} v & \text { if } n=-3, \\
u-3=0 & \text { with cofactor }-\frac{2}{3} u & \text { if } n=-3, \\
2 u-5 v=0 & \text { with cofactor }-\frac{3}{5} u+\frac{1}{2} v+1 & \text { if } n=-5 .
\end{array}
$$

Proof. We substitute

$$
\begin{aligned}
f & =a_{0}+a_{1} u+a_{2} v, \\
P & =u\left(\frac{1-n}{2 n} u+\frac{n+3}{4} v+\frac{n+7}{2}\right), \\
Q & =v\left(-u+\frac{1}{2} v+1\right) \\
K & =k_{0}+k_{1} u+k_{2} v
\end{aligned}
$$

in the definition of invariant algebraic curve (12). The polynomial that we get must have all its coefficients equal to zero, this provides a system of equations to solve whose unknowns are the coefficients of the polynomials $f$ and $K$, and also the parameter $n$. Solving this system by direct computation we find the five invariant straight lines of the statement of the lemma with their corresponding cofactors. This completes the proof of the lemma.

Lemma 6. The unique irreducible invariant algebraic curves of system (2) of degree 2 are

$$
\begin{aligned}
& (2 u-3 v)^{2}-12 u=0 \quad \text { with cofactor } 2-\frac{4}{3} u+v \quad \text { if } n=-3, \\
& 3(2 u-5 v)^{2}-20 u+150 v=0 \quad \text { with cofactor } 1-\frac{6}{5} u+v \quad \text { if } n=-5 \text {, } \\
& (u-3 v)^{2}+18 v=0 \quad \text { with cofactor } 1-\frac{7}{6} u+v \quad \text { if } n=-6 .
\end{aligned}
$$

Proof. Taking $f=a_{0}+a_{1} u+a_{2} v+a_{3} u^{2}+a_{4} u v+a_{5} v^{2}$ the proof is analogous to the proof of Lemma 5 .

Now we can prove the "if" part of Theorem 1. The difficult part is the "only if" that shall be proved later on.

Proof of the "if" part of Theorem 1. When $n \in\{-6,-5,-3,-1\}$ by Lemmas 5 and 6 system (2) has at least three invariant algebraic curves $f_{1}=0, f_{2}=0$ and $f_{3}=0$ 
with cofactors $K_{i}$ for $i=1,2,3$ given in the statement of Lemmas 5 and 6 . By the statement (iv) of Theorem 4 with $p=3, q=0$ and $m=2$ it follows that system (2) for these values of $n$ has either a first integral $f_{1}^{\lambda_{1}} f_{2}^{\lambda_{2}} f_{3}^{\lambda_{3}}$ if $\sum_{i=1}^{p} \lambda_{i} K_{i}=0$, or an integrating factor $f_{1}^{\lambda_{1}} f_{2}^{\lambda_{2}} f_{3}^{\lambda_{3}}$ if $\sum_{i=1}^{p} \lambda_{i} K_{i}=-\operatorname{div}(P, Q)$. So these systems are Liouvillian integrable.

Case 1: $n=-1$. Then taking $f_{1}=u=0, f_{2}=v=0$ and $f_{3}=2 u-3 v-6=0$ with their corresponding cofactors $K_{i}$ for $i=1,2,3$, we obtain that $K_{1}-3 K_{2}+2 K_{3}=0$. Therefore $f_{1} f_{2}^{-3} f_{3}^{2}$ is a first integral of system (2) with $n=-1$.

Case 2: $n=-3$. Again taking $f_{1}=u=0, f_{2}=v=0$ and $f_{3}=2 u-3 v-6=0$ with their corresponding cofactors $K_{i}$ for $i=1,2,3$, we obtain that $K_{1}-2 K_{2}+2 K_{3}=0$. Therefore $f_{1} f_{2}^{-2} f_{3}^{2}$ is a first integral of system (2) with $n=-3$.

Case 3: $n=-5$. Now taking $f_{1}=u=0, f_{2}=v=0$ and $f_{3}=3(2 u-5 v)^{2}-$ $20 u+150 v=0$ with their corresponding cofactors $K_{i}$ for $i=1,2,3$, we obtain that $K_{1}-3 K_{2}+2 K_{3}=0$. Therefore $f_{1} f_{2}^{-3} f_{3}^{2}$ is a first integral of system (2) with $n=-5$. Case 4: $n=-6$. We take $f_{1}=u=0, f_{2}=v=0$ and $f_{3}=(u-3 v)^{2}+18 v=0$ with their corresponding cofactors $K_{i}$ for $i=1,2,3$. Then we obtain that $-2 K_{1}+$ $\frac{5}{2} K_{2}-3 K_{3}=-\operatorname{div}(P, Q)=-(18-26 u+3 v) / 12$. Therefore $R=f_{1}^{-2} f_{2}^{5 / 2} f_{3}^{-3}$ is an integrating factor of system (2) with $n=-6$. Now computing the integral (15) we get the first integral given in the statement of Theorem 1 for $n=-6$. This completes the proof of the "if" part of Theorem 1.

From the "if" part of Theorem 1 for $n=-1,-3,-5$ the polynomial differential system (2) has irreducible invariant algebraic curves of degree 3 of the form

$$
\begin{array}{ll}
u(2 u-3 v-6)^{2}-h v^{3}=0 & \text { if } n=-1, \\
u(2 u-3 v-6)^{2}-h v^{2}=0 & \text { if } n=-3, \\
u\left(3(2 u-5 v)^{2}-20 u+150 v\right)^{2}-h v^{3}=0 & \text { if } n=-5,
\end{array}
$$

for some values of $h \in \mathbb{R}^{3}$. Note that all the solutions curves of system (2) when $n=-1,-3,-5$ are invariant algebraic curves of degree 1,2 or 3 .

Lemma 7. If $n \notin\{-1,-3,-5\}$ then the polynomial differential system (2) has no irreducible invariant algebraic curves of degree 3.

Proof. Taking $f=a_{0}+a_{1} u+a_{2} v+a_{3} u^{2}+a_{4} u v+a_{5} v^{2}+a_{6} u^{3}+a_{7} u^{2} v+a_{8} u v^{2}+a_{9} v^{3}$ the proof is analogous to the proof of Lemma 5 . In this proof we have used the program mathematica for helping us in the computations.

Lemma 8. If $n \notin\{-7,-5,-3,-1,1,3\}$ then the polynomial differential system (2) has no irreducible invariant algebraic curves of degree $k>3$.

Proof. Assume that $f=0$ is an irreducible invariant algebraic curve of degree $k>3$ of system (2), and let $f_{i}$ be the homogeneous part of degree $i$ of $f$ for $i=0,1, \ldots, k$ with $f_{k} \neq 0$. 
Let $k_{0}+k_{1} u+k_{2} v$ be the cofactor of the invariant algebraic curve $f=0$. From (2) and (12) we have that the terms of order $k+1$ in the variables $u$ and $v$ in (12) are

$$
u\left(\frac{1-n}{2 n} u+\frac{n+3}{4} v\right) \frac{\partial f_{k}}{\partial u}+v\left(-u+\frac{1}{2} v\right) \frac{\partial f_{k}}{\partial v}=\left(k_{1} u+k_{2} v\right) f_{k} .
$$

Since $f_{k}$ is a homogeneous polynomial of degree $k$ by the Euler theorem for the homogeneous functions, we obtain that

$$
u \frac{\partial f_{k}}{\partial u}+v \frac{\partial f_{k}}{\partial v}=k f_{k}
$$

Considering equations (4) and (18) as a linear system of two equations with the two unknowns $\partial f_{k} / \partial u$ and $\partial f_{k} / \partial v$, since $n \neq-1$ we get that

$$
\begin{aligned}
& \frac{\partial f_{k}}{\partial u}=\frac{2 n\left(2\left(k+k_{1}\right) u+\left(2 k_{2}-k\right) v\right)}{(n+1) u(2 u+n v)} f_{k}, \\
& \frac{\partial f_{k}}{\partial v}=\frac{n\left(n k+3 k-4 k_{2}\right) v-2\left(n k-k+2 k_{1} n\right) u}{(n+1) v(2 u+n v)} f_{k} .
\end{aligned}
$$

Solving the first differential equation of (19) we obtain that

$$
f_{k}(u, v)=g(v) u^{\frac{2\left(2 k_{2}-k\right)}{n+1}}(2 u+n v)^{\frac{2\left(k n+k-2 k_{2}+k_{1} n\right)}{n+1}},
$$

where $g(v)$ is an arbitrary function in the variable $v$; and solving the second differential equation of (19) we get that

$$
f_{k}(u, v)=h(u) v^{\frac{k-k n-2 k_{1} n}{n+1}}(2 u+n v)^{\frac{2\left(k n+k-2 k_{2}+k_{1} n\right)}{n+1}},
$$

where $h(u)$ is an arbitrary function in the variable $u$. So, from (20) and (21), since an invariant algebraic curve is determined unless a non-zero constant and since $f_{k}(u, v)$ is a homogeneous polynomial of degree $k$, we have that

$$
f_{k}(u, v)=u^{\frac{2\left(2 k_{2}-k\right)}{n+1}} v^{\frac{k-k n-2 k_{1} n}{n+1}}(2 u+n v)^{\frac{2\left(k n+k-2 k_{2}+k_{1} n\right)}{n+1}} .
$$

Of course

$$
\frac{2\left(2 k_{2}-k\right)}{n+1}+\frac{k-k n-2 k_{1} n}{n+1}+\frac{2\left(k n+k-2 k_{2}+k_{1} n\right)}{n+1}=k,
$$

and every one of the three exponents which appear in the expression of $f_{k}(u, v)$ must be a non-negative integer.

Now the terms of order $k$ in the variables $u$ and $v$ in (12) are

$$
\begin{array}{r}
u\left(\frac{1-n}{2 n} u+\frac{n+3}{4} v\right) \frac{\partial f_{k-1}}{\partial u}+v\left(-u+\frac{1}{2} v\right) \frac{\partial f_{k-1}}{\partial v}= \\
\left(k_{1} u+k_{2} v\right) f_{k-1}+k_{0} f_{k}-\frac{n+7}{2} u \frac{\partial f_{k}}{\partial u}-v \frac{\partial f_{k}}{\partial v} .
\end{array}
$$

Again since $f_{k-1}$ is a homogeneous polynomial of degree $k-1$ by the Euler theorem, we obtain that

$$
u \frac{\partial f_{k-1}}{\partial u}+v \frac{\partial f_{k-1}}{\partial v}=(k-1) f_{k-1}
$$


Considering equations (22) and (23) as a linear system of two equations with the two unknowns $\partial f_{k-1} / \partial u$ and $\partial f_{k-1} / \partial v$, since $n \neq-1$ we get that

$$
\begin{aligned}
\frac{\partial f_{k-1}}{\partial u}= & \frac{1}{(n+1)^{2}} 2 n u^{-\frac{2 k}{n+1}-1} v^{-\frac{\left(k+2 k_{1}\right) n}{n+1}}(2 u+n v)^{-\frac{4 k_{2}}{n+1}-2} \\
& \left(2 u^{\frac{4 k_{2}}{n+1}} v^{\frac{k}{n+1}}(2 u+n v)^{\frac{2\left(k+\left(k+k_{1}\right) n\right)}{n+1}}(-2 k(n(n+6)+1) u+\right. \\
& \left.4 k n v-2 n(n+5)\left(k_{1} u+k_{2} v\right)+k_{0}(n+1)(2 u+n v)\right)+ \\
& u^{\frac{2 k}{n+1}} v^{\frac{\left(k+2 k_{1}\right) n}{n+1}}(2 u+n v)^{\frac{4 k_{2}}{n+1}+1}(n+1) \\
& \left.\left(2\left(k+k_{1}-1\right) u-k v+2 k_{2} v+v\right) f(u, v)\right), \\
\frac{\partial f_{k-1}}{\partial v=} & \frac{1}{(n+1)^{2}} u^{-\frac{2 k}{n+1}} v^{-\frac{k n+2 k_{1} n+n+1}{n+1}}(2 u+n v)^{-\frac{4 k_{2}}{n+1}-2} \\
& \left(4 n u ^ { \frac { 4 k _ { 2 } } { n + 1 } } v ^ { \frac { k } { n + 1 } } ( 2 u + n v ) ^ { \frac { 2 ( k + ( k + k _ { 1 } ) n ) } { n + 1 } } \left(2 n(n+5)\left(k_{1} u+k_{2} v\right)+\right.\right. \\
& \left.2 k((n(n+6)+1) u-2 n v)-k_{0}(n+1)(2 u+n v)\right)- \\
& u^{\frac{2 k}{n+1}} v^{\frac{\left(k+2 k_{1}\right) n}{n+1}}(2 u+n v)^{\frac{4 k_{2}}{n+1}+1}(n+1) \\
& \left(2\left(-k+\left(k+2 k_{1}-1\right) n+1\right) u+\right. \\
& \left.\left.n\left(4 k_{2}+n-k(n+3)+3\right) v\right) f(u, v)\right) .
\end{aligned}
$$

Solving the first differential equation of (24) we obtain that

$$
f_{k-1}(u, v)=2^{\frac{2\left(2 k_{2}-k+1\right)}{n+1}} u^{\frac{2\left(2 k_{2}-k+1\right)}{n+1}} g(v)(2 u+n v)^{\frac{2\left(k n+k-2 k_{2}+k_{1} n-n-1\right)}{n+1}}-A(u, v),
$$

where $g(v)$ is an arbitrary function in the variable $v$, and

$$
\begin{aligned}
A(u, v)= & \frac{2 n}{n^{2}-1} u^{\frac{2\left(2 k_{2}-k\right)}{n+1}} v^{\frac{k-k n-2 k_{1} n}{n+1}}(2 u+n v) \frac{2\left(k n+k-2 k_{2}+k_{1} n-n-1\right)}{n+1} \\
& \left(4\left(-k_{0}(1+n)+k_{1} n(5+n)+k(1+n(6+n))\right) u+\right. \\
& \left.(n-1) n\left(4 k+k_{0}(1+n)-2 k_{2}(5+n)\right) v\right),
\end{aligned}
$$

recall that $n \neq \pm 1$. Solving the second differential equation of (24) we get that

$$
f_{k-1}(u, v)=h(u) v^{\frac{k-k n-2 k_{1} n+n-1}{n+1}}(2 u+n v)^{\frac{2\left(k n+k-2 k_{2}+k_{1} n-n-1\right)}{n+1}}-A(u, v),
$$

where $h(u)$ is an arbitrary function in the variable $u$.

From (25) and (26) it follows that

$$
\begin{aligned}
f_{k-1}(u, v)= & c_{k-1} 2^{\frac{2\left(2 k_{2}-k+1\right)}{n+1}} u^{\frac{2\left(2 k_{2}-k+1\right)}{n+1}} v^{\frac{k-k n-2 k_{1} n+n-1}{n+1}}(2 u+n v)^{\frac{2\left(k n+k-2 k_{2}+k_{1} n-n-1\right)}{n+1}}- \\
& A(u, v),
\end{aligned}
$$

where $c_{k-1} \in \mathbb{R}$. 
Looking at the exponents of $u$ and $v$ in $f_{k}(u, v)$ and in $f_{k-1}(u, v)$ it follows that $c_{k-1}=0$ because $n \neq 1$. So

$$
f_{k-1}(u, v)=A(u, v) .
$$

The terms of order $k-1$ in the variables $u$ and $v$ in (12) are

$$
\begin{aligned}
u\left(\frac{1-n}{2 n} u+\frac{n+3}{4} v\right) \frac{\partial f_{k-2}}{\partial u}+v\left(-u+\frac{1}{2} v\right) \frac{\partial f_{k-2}}{\partial v}= \\
\left(k_{1} u+k_{2} v\right) f_{k-2}+k_{0} f_{k-1}-\frac{n+7}{2} u \frac{\partial f_{k-1}}{\partial u}-v \frac{\partial f_{k-1}}{\partial v}
\end{aligned}
$$

Again since $f_{k-2}$ is a homogeneous polynomial of degree $k-2$ by the Euler theorem, we obtain that

$$
u \frac{\partial f_{k-2}}{\partial u}+v \frac{\partial f_{k-2}}{\partial v}=(k-2) f_{k-2} .
$$

Considering equations (4) and (4) as a linear system of two equations with the two unknowns $\partial f_{k-2} / \partial u$ and $\partial f_{k-2} / \partial v$, since $n \neq-1$ we get that

$$
\begin{aligned}
\frac{\partial f_{k-2}}{\partial u}= & \frac{2 n}{(n-1)(n+1)^{3}} u^{-\frac{2 k}{n+1}-1} v^{-\frac{\left(k+2 k_{1}\right) n}{n+1}}(2 u+n v)^{-\frac{4\left(k_{2}+n+1\right)}{n+1}} \\
& \left(4 n u^{\frac{4 k_{2}}{n+1}} v^{\frac{k}{n+1}}(2 u+n v)^{\frac{2\left(n k+k+k_{1} n\right)}{n+1}} F(u, v)-\right. \\
& (n-1)(n+1)^{2} u^{\frac{2 k}{n+1}} v^{\frac{\left(k+2 k_{1}\right) n}{n+1}}(2 u+n v)^{\frac{4 k_{2}+3 n+3}{n+1}} \\
& \left.\left(\left(k-2\left(k_{2}+1\right)\right) v-2\left(k+k_{1}-2\right) u\right) f(u, v)\right), \\
\frac{\partial f_{k-2}}{\partial v=} & \frac{1}{(n-1)(n+1)^{3}} u^{-\frac{2 k}{n+1}} v^{-\frac{k n+2 k_{1} n+n+1}{n+1}}(2 u+n v)^{-\frac{4\left(k_{2}+n+1\right)}{n+1}} \\
& \left(-8 n^{2} u^{\frac{4 k_{2}}{n+1}} v^{\frac{k}{n+1}}(2 u+n v)^{\frac{2\left(n k+k+k_{1} n\right)}{n+1}} F(u, v)-\right. \\
& (n-1)(n+1)^{2} u^{\frac{2 k}{n+1}} v^{\frac{\left(k+2 k_{1}\right) n}{n+1}}(2 u+n v)^{\frac{4 k_{2}+3 n+3}{n+1}} \\
& \left(2\left(k(n-1)+2\left(k_{1}-1\right) n+2\right) u+\right. \\
& \left.\left.n\left(2\left(2 k_{2}+n+3\right)-k(n+3)\right) v\right) f(u, v)\right),
\end{aligned}
$$


where

$$
\begin{aligned}
F(u, v)=( & 8 k^{2}\left(\left(n^{2}+6 n+1\right)^{2} u^{2}+\right. \\
& \left.n\left(n^{3}+3 n^{2}-17 n-3\right) v u-2(n-1) n^{2} v^{2}\right)- \\
& 2\left(2\left(n^{2}+6 n+1\right)\left(\left(1-4 k_{1}\right) n^{2}+\left(8-20 k_{1}\right) n+4 k_{0}(n+1)+7\right) u^{2}-\right. \\
& n\left(n^{4}+4 k_{1} n^{3}+6 n^{3}+8 k_{1} n^{2}+4 n^{2}-60 k_{1} n+26 n+\right. \\
& k_{0}\left(n^{4}+4 n^{3}-18 n^{2}-12 n+9\right)-2 k_{2}\left(n^{4}+8 n^{3}-2 n^{2}-88 n-15\right)+ \\
& \left.27) v u+2(n-1) n^{2}\left(n+2 k_{0}(n+1)-4 k_{2}(n+5)+1\right) v^{2}\right) k+ \\
& k_{0}^{2}(n+1)^{2}\left(8 u^{2}-2(n-3) n v u-(n-1) n^{2} v^{2}\right)+k_{0}(n+1) \\
& \left(-4\left(\left(4 k_{1}-1\right) n^{2}+4\left(5 k_{1}-2\right) n-7\right) u^{2}+2 n\left(\left(k_{1}-1\right) n^{3}+\right.\right. \\
& \left.\left(2 k_{1}-7\right) n^{2}-3\left(5 k_{1}+1\right) n+2 k_{2}\left(n^{2}+2 n-15\right)+3\right) v u+ \\
& \left.(n-1) n^{2}\left(-n+4 k_{2}(n+5)-1\right) v^{2}\right)+2 n(n+5)\left(4 k_{1}^{2} n(n+5) u^{2}+\right. \\
& k_{1}\left(n\left(n^{2}+4 n-2 k_{2}\left(n^{2}+2 n-15\right)+3\right) v-2\left(n^{2}+8 n+7\right) u\right) u+ \\
& \left.\left.k_{2}(n-1) v\left(2\left(n^{2}+7 n+6\right) u+n\left(n-2 k_{2}(n+5)+1\right) v\right)\right)\right) .
\end{aligned}
$$

Solving the differential equation of $\partial f_{k-2} / \partial u$ we obtain that (30)

$$
f_{k-2}(u, v)=2^{\frac{2\left(-k+2 k_{2}+2\right)}{n+1}} u^{\frac{2\left(-k+2 k_{2}+2\right)}{n+1}} g(v)(2 u+n v)^{\frac{2\left(n k+k-2 k_{2}+k_{1} n-2 n-2\right)}{n+1}}+B(u, v),
$$


where $g(v)$ is an arbitrary function in the variable $v$; and

$$
\begin{aligned}
B(u, v)= & \frac{2 n^{2}}{(n-3)\left(n^{2}-1\right)^{2}} u^{\frac{2\left(2 k_{2}-k\right)}{n+1}} v^{\frac{k-k n+2 k_{1} n}{n+1}}(2 u+n v)^{2\left(k+k_{1}-\frac{k_{1}+2 k_{2}}{n+1}-2\right)} \\
& \left(8(n-3)\left(-k n^{2}-k_{1} n^{2}-6 k n+k_{0} n-5 k_{1} n-k+k_{0}\right)\right. \\
& \left(-2 k n^{2}-2 k_{1} n^{2}+n^{2}-12 k n+2 k_{0} n-10 k_{1} n+8 n-2 k+2 k_{0}+7\right) u^{2}- \\
& 8(n-1) n\left(-k n^{4}-k k_{0} n^{4}+k_{0} n^{4}-k_{0} k_{1} n^{4}-k_{1} n^{4}+2 k k_{2} n^{4}+\right. \\
& 2 k_{1} k_{2} n^{4}-2 k_{2} n^{4}-4 k^{2} n^{3}+k_{0}^{2} n^{3}-6 k n^{3}-4 k k_{0} n^{3}+8 k_{0} n^{3}- \\
& 4 k k_{1} n^{3}-3 k_{0} k_{1} n^{3}-9 k_{1} n^{3}+16 k k_{2} n^{3}-2 k_{0} k_{2} n^{3}+14 k_{1} k_{2} n^{3}- \\
& 22 k_{2} n^{3}-12 k^{2} n^{2}-k_{0}^{2} n^{2}-4 k n^{2}+18 k k_{0} n^{2}+10 k_{0} n^{2}-8 k k_{1} n^{2}+ \\
& 13 k_{0} k_{1} n^{2}-23 k_{1} n^{2}-4 k k_{2} n^{2}-6 k_{0} k_{2} n^{2}-10 k_{1} k_{2} n^{2}-58 k_{2} n^{2}+ \\
& 68 k^{2} n-5 k_{0}^{2} n-26 k n+12 k k_{0} n+60 k k_{1} n+15 k_{0} k_{1} n-15 k_{1} n- \\
& 176 k k_{2} n+26 k_{0} k_{2} n-150 k_{1} k_{2} n+22 k_{2} n+12 k^{2}-3 k_{0}^{2}-27 k- \\
& \left.9 k k_{0}-3 k_{0}-30 k k_{2}+30 k_{0} k_{2}+60 k_{2}\right) v u+(n-3)(n-1)^{2} n^{2} \\
& \left.\left(4 k+k_{0}-10 k_{2}+k_{0} n-2 k_{2} n\right)\left(4 k+k_{0}-10 k_{2}+k_{0} n-2 k_{2} n+n+1\right) v^{2}\right) .
\end{aligned}
$$

Recall that $n \notin\{-1,1,3\}$.

Solving the differential equation of $\partial f_{k-2} / \partial v$ we get that

$$
f_{k-2}(u, v)=h(u) v^{\frac{k-k n-2 k_{1} n+2 n-2}{n+1}}(2 u+n v)^{\frac{2\left(k+k n-2 k_{2}+k_{1} n-2 n-2\right)}{n+1}},
$$

where $h(u)$ is an arbitrary function in the variable $u$. From (30) and (31) it follows that

$$
\begin{aligned}
f_{k-2}(u, v)= & c_{k-2} 2^{\frac{2\left(2 k_{2}-k+2\right)}{n+1}} u^{\frac{2\left(2 k_{2}-k+2\right)}{n+1}} v^{\frac{k-k n-2 k_{1} n+2 n-2}{n+1}}(2 u+n v)^{\frac{2\left(k+k n-2 k_{2}+k_{1} n-2 n-2\right)}{n+1}} \\
& +B(u, v),
\end{aligned}
$$

where $c_{k-2} \in \mathbb{R}$.

Looking at the exponents of $u$ and $v$ in $f_{k}(u, v)$ and in $f_{k-2}(u, v)$ it follows that $c_{k-2}=0$ because $n \neq 1$. So

$$
f_{k-2}(u, v)=B(u, v) .
$$

Looking at $f_{k}, f_{k-1}$ and $f_{k-2}$ we see that all of them have the factor

$$
g(u, v)=u^{\frac{2\left(2 k_{2}-k\right)}{n+1}} v^{\frac{k-k n-2 k_{1} n}{n+1}} .
$$

It is easy to check by induction that all the $f_{i}$ for $i=k, k-1, \ldots$ with $f_{i} \neq 0$ have the same factor $g(u, v)$, so $g(u, v)$ divides $f(u, v)$. Since we are looking for irreducible 
invariant algebraic curves $f(u, v)=0$, and $u=0$ and $v=0$ are invariant algebraic curves, we must have

$$
\frac{2\left(2 k_{2}-k\right)}{n+1}=0, \quad \frac{k-k n-2 k_{1} n}{n+1}=0 \quad \text { and } \quad \frac{2\left(k n+k-2 k_{2}+k_{1} n\right)}{n+1}=k .
$$

Therefore

In short we have

$$
k_{1}=\frac{k(1-n)}{2 n}, \quad k_{2}=\frac{k}{2} .
$$

$$
\begin{aligned}
f_{k}(u, v)= & (2 u+n v)^{k}, \\
f_{k-1}(u, v)= & \frac{2 n}{n-1}(2 u+n v)^{k-2}\left(\left(k-k_{0}\right)(n-1) n v-2\left(k(n+7)-2 k_{0}\right) u\right), \\
f_{k-2}(u, v)= & \frac{2 n^{2}}{(n-3)(n-1)^{2}}(2 u+n v)^{k-4}\left(k^{2}(n-3)((n-1) n v-2(n+7) u)^{2}+\right. \\
& k_{0}\left(8(n-3)\left(2 k_{0}+n+7\right) u^{2}-8(n-1) n\left(k_{0}(n-3)+n(n+6)-3\right) v u+\right. \\
& \left.\left(k_{0}+1\right)(n-3)(n-1)^{2} n^{2} v^{2}\right)-k\left(4(n-3)(n+7)\left(4 k_{0}+n+7\right) u^{2}-\right. \\
& 4(n-1) n\left(k_{0}(n-3)(n+9)+n(3 n+20)+9\right) v u+ \\
& \left.\left.\left(2 k_{0}+1\right)(n-3)(n-1)^{2} n^{2} v^{2}\right)\right) .
\end{aligned}
$$

Now we consider equation (12) restricted to $u=0$, i.e.

$$
\left(\frac{v^{2}}{2}+v\right) F^{\prime}(v)=\left(k_{0}+\frac{k v}{2}\right) F(v) \text {, }
$$

where $F(v)=f(0, v)$. Solving this differential equation we obtain $F(v)=c v^{k_{0}}(2+$ $v)^{k-k_{0}}$ where $c \in \mathbb{R}$. Since $F(v)$ must be a polynomial of degree at most $k$ it follows that $k_{0}$ is an integer of $\{0,1, \ldots, k\}$. Since $f_{k}(u, v)=(2 u+n v)^{k}$ we get that $c=n^{k}$. Therefore

$$
f(u, v)=n^{k} v^{k_{0}}(2+v)^{k-k_{0}}+u g(u, v) .
$$

On the other hand equation (12) restricted to $v=0$ is

$$
\left(\frac{(1-n) u^{2}}{2 n}+\frac{1}{2}(n+7) u\right)\left(G(u)+u G^{\prime}(u)\right)=u\left(k_{0}+\frac{k(1-n) u}{2 n}\right) G(u),
$$

where $G(u)=g(u, 0)$. Since $n \neq-7$ solving this differential equation we obtain

$$
G(u)=d u^{\frac{2 k_{0}}{n+7}-1}((n-1) u-n(n+7))^{k-\frac{2 k_{0}}{n+7}},
$$

with $d \in \mathbb{R}$. Since $u G(u)$ must be a polynomial of degree at most $k$ it follows that $\frac{2 k_{0}}{n+7}$ is an integer of $\{0,1, \ldots, k\}$. Again since $f_{k}(u, v)=(2 u+n v)^{k}$ we get that $d=\left(\frac{2}{n-1}\right)^{k}$. So

$$
f(u, v)=n^{k} v^{k_{0}}(2+v)^{k-k_{0}}+\left(\frac{2}{n-1}\right)^{k} u^{\frac{2 k_{0}}{n+7}}((n-1) u-n(n+7))^{k-\frac{2 k_{0}}{n+7}}+u v h(u, v) .
$$


Assume that $k_{0}=0$. Then, substituting $f(u, v)$ in $u\left(\frac{1-n}{2 n} u+\frac{n+3}{4} v+\frac{n+7}{2}\right) \frac{\partial f}{\partial u}+v\left(-u+\frac{1}{2} v+1\right) \frac{\partial f}{\partial v}=\left(\frac{k(1-n)}{2 n} u+\frac{k}{2} v\right) f$, and evaluating this expression first on $u=0$ and after on $v=0$ we obtain

$$
\begin{aligned}
& 2^{k-1} k\left(\frac{n(n+7)}{n-1}\right)^{k} v \equiv 0, \\
& 2^{k-1} k(1-n) n^{k-1} u \equiv 0,
\end{aligned}
$$

respectively. Since this system has no solution, it follows that $k_{0}$ cannot be zero. Hence $k_{0}$ and $\frac{2 k_{0}}{n+7}$ belong to $\{1,2, \ldots, k\}$.

The terms of order 0 in the variables $u$ and $v$ in (12), are $k_{0} f_{0}=0$. Since $k_{0} \neq 0$ we get that $f_{0}=0$.

The terms of order 1 in the variables $u$ and $v$ in (12) are

$$
\frac{n+7}{2} u \frac{\partial f_{1}}{\partial u}+v \frac{\partial f_{1}}{\partial v}=k_{0} f_{1} \text {. }
$$

Solving this linear partial differential equation we obtain that

$$
f_{1}(u, v)=c_{1} u^{\frac{2 k_{0}}{n+7}} F\left(u^{-\frac{2}{n+7}} v\right),
$$

where $F$ is an arbitrary function of the variable $u^{-\frac{2}{n+7}} v$. If $c_{1} \neq 0$, since $f_{1}$ must be a homogeneous polynomial of degree 1 , we obtain

$$
f_{1}(u, v)=c_{1} u^{\frac{2 k_{0}-2 m}{n+7}} v^{m}
$$

with $m \in\{0,1\}$ such that

$$
\frac{2 k_{0}-2 m}{n+7}+m=1
$$

Therefore, either $m=0$ and $k_{0}=(n+7) / 2$, or $m=1$ and $k_{0}=1$. But this last case is not possible because $\frac{2 k_{0}}{n+7}=\frac{2}{n+7}$ must belong to $\{1,2, \ldots, k\}$, and this only can occur if $n=-5$, and this case is excluded by assumptions. In short we distinguish two cases.

Case 1: $c_{1} \neq 0, m=0$ and $k_{0}=(n+7) / 2$. Then $f_{1}=c_{1} u$. The terms of order $2 \mathrm{in}$ the variables $u$ and $v$ in (12) are

$$
u\left(\frac{1-n}{2 n} u+\frac{n+3}{4} v\right) c_{1}+\frac{n+7}{2} u \frac{\partial f_{2}}{\partial u}+v \frac{\partial f_{2}}{\partial v}=\frac{n+7}{2} f_{2}+\left(\frac{k(1-n)}{2 n} u+\frac{k}{2} v\right) f_{1} .
$$

Solving this linear partial differential equation we get

$$
f_{2}(u, v)=-\frac{c_{1} u(4(k-1)(n-1) u+n(7+n)(3-2 k+n) v)}{4 n(n+7)}+c_{2} u G\left(u^{-\frac{2}{n+7}} v\right),
$$

where $G$ is an arbitrary function of the variable of the variable $u^{-\frac{2}{n+7}} v$. If $c_{2} \neq 0$ since $f_{2}$ must be a homogeneous polynomial of degree 2 it follows that

$$
G\left(u^{-\frac{2}{n+7}} v\right)=c_{2} u^{-\frac{2 m}{n+7}} v^{m}
$$


with $m=0,1$ and

$$
-\frac{2 m}{n+7}+m=1 \text {. }
$$

But this is not possible so $c_{2}=0$, and $f_{2}$ as $f_{1}$ is divisible by $u$. Repeating these arguments for $f_{\ell}$ with $\ell=2, \ldots, k$ we get that $f_{\ell}$ also is divisible by $u$ in contradiction with the fact that $f_{k}=(2 u+n v)^{k}$.

Case 2: $c_{1}=0$. Then $f_{0}=f_{1}=0$. Now the terms of order 2 in the variables $u$ and $v$ in $(12)$ are

$$
\frac{n+7}{2} u \frac{\partial f_{2}}{\partial u}+v \frac{\partial f_{2}}{\partial v}=k_{0} f_{2}
$$

which coincides with equation (4), and its solution is given in (4), i.e.

$$
f_{2}(u, v)=c_{2} u^{\frac{2 k_{0}-2 m}{n+7}} v^{m}
$$

and if $c_{2} \neq 0$ then $m \in\{0,1,2\}$ such that

$$
\frac{2 k_{0}-2 m}{n+7}+m=2 .
$$

Therefore, either $m=0$ and $k_{0}=n+7$, or $m=1$ and $k_{0}=(n+9) / 2$, or $m=2$ and $k_{0}=2$. But these last two cases are not possible because $\frac{2 k_{0}}{n+7}$ must belong to $\{1,2, \ldots, k\}$, and in the first $\frac{2 k_{0}}{n+7}=(n+9) /(n+7)$ and in the second only can occur if $n=-3$, and this case is excluded by assumptions. In short we distinguish two cases.

Case 3: $c_{2} \neq 0, m=0$ and $k_{0}=n+7$.

Case 4: $c_{2}=0$.

The rest of the proof follows in a similar way to the Cases 1 and 2 .

From the results of Lemmas 5 to 8 in order to know all the invariant algebraic curves of system (2) only remains to show that system (2) with $n \in\{-7,1,3\}$ has no invariant algebraic curves of degree $k>3$. This will be proved in the next tree lemmas.

Lemma 9. If $n=1$ then the polynomial differential system (2) has no irreducible invariant algebraic curves of degree $k>3$.

Proof. We work as in the proof of Lemma 8 but now with $n=1$. Let $k_{0}+k_{1} u+k_{2} v$ be the cofactor of the invariant algebraic curve $f=0$. From (2) and (12) we have that the terms of order $k+1$ in (12) are

$$
u v \frac{\partial f_{k}}{\partial u}+v\left(-u+\frac{1}{2} v\right) \frac{\partial f_{k}}{\partial v}=\left(k_{1} u+k_{2} v\right) f_{k} .
$$

Solving this linear partial differential equation and since $f_{k}$ is a homogeneous polynomial of degree $k$ we get that

$$
f_{k}=u^{-k+2 k_{2}} v^{-k_{1}}(2 u+v)^{2 k+k_{1}-2 k_{2}} .
$$

Of course

$$
-k+2 k_{2}-k_{1}+2 k+k_{1}-2 k_{2}=k
$$


and every one of the three exponents which appear in the expression of $f_{k}$ must be a non-negative integer.

Now the terms of order $k$ in (12) are

$$
u v \frac{\partial f_{k-1}}{\partial u}+v\left(-u+\frac{1}{2} v\right) \frac{\partial f_{k-1}}{\partial v}=\left(k_{1} u+k_{2} v\right) f_{k-1}+k_{0} f_{k}-4 u \frac{\partial f_{k}}{\partial u}-v \frac{\partial f_{k}}{\partial v} .
$$

Again since $f_{k-1}$ is a homogeneous polynomial of degree $k-1$ by the Euler theorem, we obtain that

$$
u \frac{\partial f_{k-1}}{\partial u}+v \frac{\partial f_{k-1}}{\partial v}=(k-1) f_{k-1} .
$$

Considering these two previos equations as a linear system of two equations with the two unknowns $\partial f_{k-1} / \partial u$ and $\partial f_{k-1} / \partial v$, we get that

$$
\begin{aligned}
\frac{\partial f_{k-1}}{\partial u}= & u^{-k+2 k_{2}-1}(2 u+v)^{2 k+k_{1}-2 k_{2}-2}\left(4\left(-4 k+k_{0}-3 k_{1}\right) u+2\left(2 k+k_{0}-6 k_{2}\right) v\right) v^{-k_{1}}+ \\
& \frac{\left(2\left(k+k_{1}-1\right) u+\left(-k+2 k_{2}+1\right) v\right) f(u, v)}{u(2 u+v)}, \\
\frac{\partial f_{k-1}}{\partial v}= & u^{2 k_{2}-k v^{-k_{1}-1}(2 u+v)^{2 k+k_{1}-2 k_{2}-2}\left(4\left(4 k-k_{0}+3 k_{1}\right) u-2\left(2 k+k_{0}-6 k_{2}\right) v\right)} \\
& -\frac{2\left(k_{1} u+\left(-k+k_{2}+1\right) v\right) f(u, v)}{v(2 u+v)} .
\end{aligned}
$$

Solving the differential equation for $\partial f_{k-1} / \partial u$ we obtain that

$$
\begin{aligned}
f_{k-1}= & 2 u^{2 k_{2}-k} v^{-k_{1}}(2 u+v)^{2 k+k_{1}-2 k_{2}-2}\left(2^{2 k_{2}-k} u g(v) v^{k_{1}}+\right. \\
& \left.\left(-2 k-k_{0}+6 k_{2}\right) v+2\left(-4 k+k_{0}-3 k_{1}\right) u \log u\right),
\end{aligned}
$$

where $g(v)$ is an arbitrary function in the variable $v$. Solving the differential equation for $\partial f_{k-1} / \partial v$ we get that

$$
\begin{aligned}
f_{k-1}= & u^{-k} v^{-k_{1}}(2 u+v)^{2 k+k_{1}-2 k_{2}-2}\left(h(u) u^{k}+2\left(\left(-2 k-k_{0}+6 k_{2}\right) v+\right.\right. \\
& \left.\left.2\left(4 k-k_{0}+3 k_{1}\right) u \log v\right) u^{2 k_{2}}\right),
\end{aligned}
$$

where $h(u)$ is an arbitrary function in the variable $u$.

Since $f_{k-1}$ must be a homogeneous polynomial of degree $k-1$ from (34) and (35) it follows that

$$
k_{0}=4 k+3 k_{1},
$$

and

$$
\begin{aligned}
f_{k-1}= & -6\left(2 k+k_{1}-2 k_{2}\right) u^{2 k_{2}-k} v^{1-k_{1}}(2 u+v)^{2 k+k_{1}-2 k_{2}-2} \\
& c_{k-1} 2^{-k+2 k_{2}+1} u^{-k+2 k_{2}+1} v^{-k_{1}}(2 u+v)^{2 k+k_{1}-2 k_{2}-2},
\end{aligned}
$$

where $c_{k-1} \in \mathbb{R}$. 
We consider the terms of order $k-1$ in (12) satisfying

$$
\begin{aligned}
u v \frac{\partial f_{k-2}}{\partial u}+v\left(-u+\frac{1}{2} v\right) \frac{\partial f_{k-2}}{\partial v} & = \\
& \left(k_{1} u+k_{2} v\right) f_{k-2}+\left(4 k+3 k_{1}\right) f_{k-1}-4 u \frac{\partial f_{k-1}}{\partial u}-v \frac{\partial f_{k-1}}{\partial v}
\end{aligned}
$$

Again since $f_{k-2}$ is a homogeneous polynomial of degree $k-2$ by the Euler theorem, we obtain that

$$
u \frac{\partial f_{k-2}}{\partial u}+v \frac{\partial f_{k-2}}{\partial v}=(k-2) f_{k-2} .
$$

Considering these two previous equations as a linear system of two equations with the two unknowns $\partial f_{k-2} / \partial u$ and $\partial f_{k-2} / \partial v$, we get that

$$
\begin{gathered}
\frac{\partial f_{k-2}}{\partial u}=2^{-k} u^{-k-1} v^{-k_{1}}(2 u+v)^{-2\left(k_{2}+2\right)}\left(2 ^ { k } v ^ { k _ { 1 } } ( 2 u + v ) ^ { 2 k _ { 2 } + 3 } \left(2\left(k+k_{1}-2\right) u+\right.\right. \\
\left.\left(-k+2 k_{2}+2\right) v\right) f(u, v) u^{k}+4(2 u+v)^{2 k+k_{1}}\left(c _ { k - 1 } u \left(3 \cdot 4^{k_{2}} k_{1} v+\right.\right. \\
\left.2^{2 k_{2}+1}\left(4 u+\left(3 k-3 k_{2}-1\right) v\right)\right)-3 \cdot 2^{k}\left(2 k+k_{1}-2 k_{2}\right) v \\
\left.\left.\left(14 u+\left(6 k+3 k_{1}-6 k_{2}+1\right) v\right)\right) u^{2 k_{2}}\right), \\
\frac{\partial f_{k-2}}{\partial v}=2^{1-k} u^{-k} v^{-k_{1}-1}(2 u+v)^{-2\left(k_{2}+2\right)}\left(2 u^{2 k_{2}}(2 u+v)^{2 k+k_{1}}\right. \\
\left(3 \cdot 2^{k}\left(2 k+k_{1}-2 k_{2}\right) v\left(14 u+\left(6 k+3 k_{1}-6 k_{2}+1\right) v\right)+\right. \\
\left.c_{k-1} u\left(2^{2 k_{2}+1}\left(-4 u-3 k v+3 k_{2} v+v\right)-3 \cdot 4^{k_{2}} k_{1} v\right)\right)- \\
\left.2^{k} u^{k} v^{k_{1}}(2 u+v)^{2 k_{2}+3}\left(k_{1} u+\left(-k+k_{2}+2\right) v\right) f(u, v)\right)
\end{gathered}
$$

Solving the differential equation of $\partial f_{k-2} / \partial u$ we obtain that

$$
\begin{aligned}
f_{k-2}= & 2^{1-k} u^{2 k_{2}-k} v^{-k_{1}}(2 u+v)^{2 k+k_{1}-2 k_{2}-4}\left(2^{2 k_{2}+1} u^{2} v^{k_{1}} g(v)+\right. \\
& \left(2^{2 k_{2}+1} c_{k-1}\left(-6 k-3 k_{1}+6 k_{2}+2\right) u+3 \cdot 2^{k}\left(2 k+k_{1}-2 k_{2}\right)\right. \\
& \left.\left.\left(28 u+\left(6 k+3 k_{1}-6 k_{2}+1\right) v\right)\right) v+4^{k_{2}+2} c_{k-1} u^{2} \log u\right),
\end{aligned}
$$


where $g(v)$ is an arbitrary function in the variable $v$. Solving the differential equation of $\partial f_{k-2} / \partial v$ we get that

$$
\begin{aligned}
f_{k-2}= & v^{-k_{1}}(2 u+v)^{2 k+k_{1}-2 k_{2}-4}\left(2 ^ { 1 - k } \left(v \left(2^{2 k_{2}+1} c_{k-1}\right.\right.\right. \\
& \left(-6 k-3 k_{1}+6 k_{2}+2\right) u+3 \cdot 2^{k}\left(2 k+k_{1}-2 k_{2}\right) \\
& \left.\left(28 u+\left(6 k+3 k_{1}-6 k_{2}+1\right) v\right)\right)- \\
& \left.\left.4^{k_{2}+2} c_{k-1} u^{2} \log v\right) u^{2 k_{2}-k}+h(u)\right)
\end{aligned}
$$

where $h(u)$ is an arbitrary function in the variable $u$. From (36) and (37) it follows that $c_{k-1}=0$ and

$$
\begin{aligned}
f_{k-2}= & 6\left(2 k+k_{1}-2 k_{2}\right) u^{2 k_{2}-k} v^{1-k_{1}}(2 u+v)^{2 k+k_{1}-2 k_{2}-4} \\
& \left(28 u+\left(6 k+3 k_{1}-6 k_{2}+1\right) v\right)+ \\
& c_{k-2} 2^{-k+2 k_{2}+2} u^{-k+2 k_{2}+2} v^{-k_{1}}(2 u+v)^{2 k+k_{1}-2 k_{2}-4},
\end{aligned}
$$

where $c_{k-2} \in \mathbb{R}$.

We consider the terms of order $k-2$ in (12) satisfying

$$
\begin{aligned}
& u v \frac{\partial f_{k-3}}{\partial u}+v\left(-u+\frac{1}{2} v\right) \frac{\partial f_{k-3}}{\partial v}= \\
&\left(k_{1} u+k_{2} v\right) f_{k-3}+\left(4 k+3 k_{1}\right) f_{k-2}-4 u \frac{\partial f_{k-2}}{\partial u}-v \frac{\partial f_{k-2}}{\partial v}
\end{aligned}
$$

Again since $f_{k-3}$ is a homogeneous polynomial of degree $k-3$ by the Euler theorem, we obtain that

$$
u \frac{\partial f_{k-3}}{\partial u}+v \frac{\partial f_{k-3}}{\partial v}=(k-3) f_{k-3}
$$

Considering these two previous equations as a linear system of two equations with the two unknowns $\partial f_{k-3} / \partial u$ and $\partial f_{k-3} / \partial v$, we get that

$$
\begin{aligned}
\frac{\partial f_{k-3}}{\partial u}= & 2^{-k} u^{-k-1} v^{-k_{1}}(2 u+v)^{-2\left(k_{2}+3\right)}\left(2 ^ { k } v ^ { k _ { 1 } } ( 2 u + v ) ^ { 2 k _ { 2 } + 5 } \left(2\left(k+k_{1}-3\right) u+\right.\right. \\
& \left.\left(-k+2 k_{2}+3\right) v\right) f(u, v) u^{k}+4(2 u+v)^{2 k+k_{1}}\left(2^{2 k_{2}+1} c_{k-2}(16 u+\right. \\
& \left.\left(6 k+3 k_{1}-6 k_{2}-4\right) v\right) u^{2}+3 \cdot 2^{k}\left(2 k+k_{1}-2 k_{2}\right) v\left(616 u^{2}+\right. \\
& \left.\left.\left.168\left(2 k+k_{1}-2 k_{2}\right) v u+\left(6 k+3 k_{1}-6 k_{2}+1\right)\left(6 k+3 k_{1}-6 k_{2}+2\right) v^{2}\right)\right) u^{2 k_{2}}\right),
\end{aligned}
$$




$$
\begin{aligned}
\frac{\partial f_{k-3}}{\partial v}= & 2^{1-k} u^{-k} v^{-k_{1}-1}(2 u+v)^{-2\left(k_{2}+3\right)}\left(-2^{k} v^{k_{1}}(2 u+v)^{2 k_{2}+5}\left(k_{1} u+\right.\right. \\
& \left.\left(-k+k_{2}+3\right) v\right) f(u, v) u^{k}-2(2 u+v)^{2 k+k_{1}}\left(2^{2 k_{2}+1} c_{k-2}(16 u+\right. \\
& \left.\left(6 k+3 k_{1}-6 k_{2}-4\right) v\right) u^{2}+3 \cdot 2^{k}\left(2 k+k_{1}-2 k_{2}\right) v\left(616 u^{2}+\right. \\
& \left.\left.\left.168\left(2 k+k_{1}-2 k_{2}\right) v u+\left(6 k+3 k_{1}-6 k_{2}+1\right)\left(6 k+3 k_{1}-6 k_{2}+2\right) v^{2}\right)\right) u^{2 k_{2}}\right) .
\end{aligned}
$$

Solving the differential equation of $\partial f_{k-3} / \partial u$ we obtain that

$$
\begin{aligned}
f_{k-3}= & 2^{2-k} u^{2 k_{2}-k} v^{-k_{1}}(2 u+v)^{2 k+k_{1}-2 k_{2}-6}\left(2^{2 k_{2}+1} u^{3} g(v) v^{k_{1}}+\left(c_{k-2} 2^{2 k_{2}+1}\right.\right. \\
& \left(-6 k-3 k_{1}+6 k_{2}+4\right) u^{2}-2^{k}\left(2 k+k_{1}-2 k_{2}\right)\left(1848 u^{2}+\right. \\
& 252\left(2 k+k_{1}-2 k_{2}\right) v u+\left(6 k+3 k_{1}-6 k_{2}+1\right) \\
& \left.\left.\left.\left(6 k+3 k_{1}-6 k_{2}+2\right) v^{2}\right)\right) v+c_{k-2} 2^{2 k_{2}+5} u^{3} \log u\right)
\end{aligned}
$$

where $g(v)$ is an arbitrary function in the variable $v$. Solving the differential equation of $\partial f_{k-3} / \partial v$ we get that

$$
\begin{aligned}
f_{k-3}= & 2^{-k} u^{-k} v^{-k_{1}}(2 u+v)^{2 k+k_{1}-2 k_{2}-6}\left(2^{k} u^{k} h(u)-4 u^{2 k_{2}}\right. \\
& \left(c_{k-2} 2^{2 k_{2}+5} u^{3} \log v+v\left(2 ^ { k } ( 2 k + k _ { 1 } - 2 k _ { 2 } ) \left(1848 u^{2}+\right.\right.\right. \\
& 252\left(2 k+k_{1}-2 k_{2}\right) v u+\left(6 k+3 k_{1}-6 k_{2}+1\right) \\
& \left.\left.\left.\left.\left(6 k+3 k_{1}-6 k_{2}+2\right) v^{2}\right)-c_{k-2} 2^{2 k_{2}+1}\left(-6 k-3 k_{1}+6 k_{2}+4\right) u^{2}\right)\right)\right),
\end{aligned}
$$

where $h(u)$ is an arbitrary function in the variable $u$. From (38) and (39) it follows that $c_{k-2}=0$ and

$$
\begin{aligned}
f_{k-3}= & -4\left(2 k+k_{1}-2 k_{2}\right) u^{2 k_{2}-k} v^{1-k_{1}}(2 u+v)^{2 k+k_{1}-2 k_{2}-6}\left(1848 u^{2}+\right. \\
& \left.252\left(2 k+k_{1}-2 k_{2}\right) v u+\left(6 k+3 k_{1}-6 k_{2}+1\right)\left(6 k+3 k_{1}-6 k_{2}+2\right) v^{2}\right)+ \\
& c_{k-3} 2^{-k+2 k_{2}+3} u^{-k+2 k_{2}+3} v^{-k_{1}}(2 u+v)^{2 k+k_{1}-2 k_{2}-6},
\end{aligned}
$$

where $c_{k-3} \in \mathbb{R}$. Following this process we obtain that $c_{k-3}=0$. 
In short, we have

$$
\begin{aligned}
f_{k}= & u^{-k+2 k_{2}} v^{-k_{1}}(2 u+v)^{2 k+k_{1}-2 k_{2}}, \\
f_{k-1}= & -6\left(2 k+k_{1}-2 k_{2}\right) u^{2 k_{2}-k} v^{1-k_{1}}(2 u+v)^{2 k+k_{1}-2 k_{2}-2}, \\
f_{k-2}= & 6\left(2 k+k_{1}-2 k_{2}\right) u^{2 k_{2}-k} v^{1-k_{1}}(2 u+v)^{2 k+k_{1}-2 k_{2}-4}\left(28 u+\left(6 k+3 k_{1}-6 k_{2}+1\right) v\right), \\
f_{k-3}= & -4\left(2 k+k_{1}-2 k_{2}\right) u^{2 k_{2}-k} v^{1-k_{1}}(2 u+v)^{2 k+k_{1}-2 k_{2}-6}\left(1848 u^{2}+\right. \\
& \left.252\left(2 k+k_{1}-2 k_{2}\right) v u+\left(6 k+3 k_{1}-6 k_{2}+1\right)\left(6 k+3 k_{1}-6 k_{2}+2\right) v^{2}\right),
\end{aligned}
$$

Repeating these arguments we shall obtain that $f=f_{0}+f_{1}+\ldots+f_{k}$ is divisible by $u^{-k+2 k_{2}} v^{-k_{1}}$, but this is not possible because $f$ is irreducible, so

$$
k_{1}=0, \quad k_{2}=k / 2 .
$$

Consequently $k_{0}=4 k$, and

$$
\begin{aligned}
f_{k} & =(2 u+v)^{k}, \\
f_{k-1} & =-6 k v(2 u+v)^{k-2}, \\
f_{k-2} & =6 k v(2 u+v)^{k-4}(28 u+(1+3 k) v), \\
f_{k-3} & =-4 k v(2 u+v)^{k-6}\left(1848 u^{2}+252 k u v+(1+3 k)(2+3 k) v^{2}\right) .
\end{aligned}
$$

On the other hand, the terms of degree 0 in $(12)$ are $k_{0} f_{0}=0$. Therefore $f_{0}=0$. Now the terms of degree 1 in (12) are

$$
4 u \frac{\partial f_{1}}{\partial u}+v \frac{\partial f_{1}}{\partial v}=4 k f_{1}
$$

Solving this linear partial differential equation we obtain that

$$
f_{1}(u, v)=u^{k} F\left(\frac{v}{\sqrt[4]{u}}\right)
$$

where $F$ is an arbitrary function of the variable $\frac{v}{\sqrt[4]{u}}$. If $F \neq 0$, since $f_{1}$ must be a homogeneous polynomial of degree 1 , we obtain

$$
f_{1}(u, v)=c_{1} u^{k-m / 4} v^{m}
$$

where $k-m / 4+m=1$. So $m=4(1-k) / 3$ and

$$
f_{1}(u, v)=c_{1} u^{\frac{k-1}{3}+k} v^{\frac{4(1-k)}{3}} .
$$

If $c_{1} \neq 0$, since $k>3$ this is a contradiction with the fact that $f_{1}$ is a homogeneous polynomial of degree 1 . So $c_{1}=0$, i.e. $f_{1}=0$. Then repeating the arguments for computing $f_{1}$ knowing that $f_{0}=0$, but now for computing $f_{2}$ knowing that $f_{1}=0$, we shall get that $f_{2}=0$, and so on. In contradiction that $f_{k}=(2 u+v)^{k}$. Hence system (2) with $n=1$ has no invariant algebraic curves of degree $k>3$.

Lemma 10. If $n=-7$ or $n=3$ then the polynomial differential system (2) has no irreducible invariant algebraic curves of degree $k>3$.

Proof. The proof follows using similar arguments to the proof of Lemma 9. 
5. The EXPONEntial FACTORS OF System (2)

As we have defined in section 2 a polynomial differential system (11) is Liouvillian integrable if it has a Darboux function

$$
f_{1}^{\lambda_{1}} \ldots f_{p}^{\lambda_{p}}\left(\exp \left(\frac{g_{1}}{h_{1}}\right)\right)^{\mu_{1}} \ldots\left(\exp \left(\frac{g_{q}}{h_{q}}\right)\right)^{\mu_{q}}
$$

as first integral or as integrating factor, where $f_{i}=0$ for $i=1, \ldots, p$ are invariant algebraic curves of system (11), and $\exp \left(g_{j} / h_{j}\right)$ for $j=1, \ldots, q$ are exponential factors of system (11). Therefore if we control all the invariant algebraic curves and all the exponential factors of a polynomial differential system we can decide if it is or not Liouvillian integrable.

We already have proved in section 3 that the Lotka-Volterra differential system (2) is Liouvillian integrable when $n \in\{-6,-5,-3,-1\}$. Now we shall prove that when $n \notin\{-6,-5,-3,-1\}$ then system (2) is not Liouvillian integrable. This will prove the "only if" part of Theorem 1 . We shall need the following lemma.

Lemma 11. If $n \notin\{-6,-5,-3,-1\}$ then system (2) has no exponential factors.

Proof. From Lemmas 5 to 10 it follows that when $n \notin\{-6,-5,-3,-1\}$ the unique invariant algebraic curves of system (2) are $u=0$ and $v=0$. By Proposition 3 for proving that there are no exponential factors coming from the invariant straight lines $u=0$ or $v=0$ it is sufficient to show that the multiplicity of these invariant straight lines is 1 . So, according with section 2 we must see that $u$ and $v$ divide the extactic polynomial

$$
e(u, v)=\left|\begin{array}{ccc}
1 & u & v \\
0 & P & Q \\
0 & P P_{x}+Q P_{y} & P Q_{x}+Q Q_{y}
\end{array}\right|,
$$

and $u^{2}$ and $v^{2}$ do not divide it, where $P=P(u, v)$ and $Q=Q(u, v)$ are the polynomials defined in (2). Since

$$
\begin{aligned}
e(u, v)=-\frac{1}{32 n^{2}} u v & \left(8(n-1)(n+1) u^{3}+4(n-2)(n-1)(n+1) u^{2} v-\right. \\
& 2(n-5) n(n+1) u v^{2}+n^{2}(n+1)(n+3) v^{3}- \\
& 8(n+1)\left(n^{2}+9 n-2\right) u^{2}+96 n(n+1) u v+ \\
& 2 n^{2}(n+1)(3 n+17) v^{2}+8 n\left(n^{2}+12 n+19\right) u+ \\
& \left.4 n^{2}(n+7)(3 n+7) v+8 n^{2}(n+5)(n+7)\right)
\end{aligned}
$$

if $(n+5)(n+7) \neq 0$ then clearly $u$ and $v$ divide $e(u, v)$, and $u^{2}$ and $v^{2}$ do not divide $e(u, v)$. By assumptions $n \neq-5$, and since for $n=-7$ we have that

$$
e(u, v)=-\frac{u v}{196}\left(48 u^{3}-216 u^{2} v+126 u v^{2}+147 v^{3}-96 u^{2}+504 u v+294 v^{2}+112 u\right),
$$

again $u^{2}$ and $v^{2}$ do not divide $e(u, v)$. Hence the multiplicity of the invariant straight lines $u=0$ and $v=0$ is 1 . 
Now from Proposition 3 it only remains to show that the multiplicity of the invariant straight line of the infinity is 1 , or equivalently that there are no exponential factors of the form $F=e^{f(u, v)}$ being $f(u, v)$ a polynomial. Assume that $F=e^{f(u, v)}$ is an exponential factor with cofactor $\ell_{0}+\ell_{1} u+\ell_{2} v$ and that the degree of the polynomial $f(u, v)$ is $k \geq 1$. Then, from (13) we have that $u(2 u+n(-2 u+3 v+n(v+2)+14)) \frac{\partial f}{\partial u}+2 n v(-2 u+v+2) \frac{\partial f}{\partial v}-4 n\left(\ell_{0}+\ell_{1} u+\ell_{2} v\right)=0$,

The terms of this polynomial of degree $k+1$ are

$$
u(2 u+n(-2 u+3 v+n v)) \frac{\partial f_{k}}{\partial u}+2 n v(-2 u+v) \frac{\partial f_{k}}{\partial v}=0,
$$

where $f_{k}$ is the homogeneous part of $f$ of degree $k$. By the Euler Theorem of homogeneous function we have that

$$
u \frac{\partial f_{k}}{\partial u}+v \frac{\partial f_{k}}{\partial v}=k f_{k}
$$

Solving these last two equations as a linear system in the unknowns $\partial f_{k} / \partial u$ and $\partial f_{k} / \partial v$, we obtain

$$
\begin{aligned}
\frac{\partial f_{k}}{\partial u} & =\frac{2 k n(2 u-v) f(u, v)}{(n+1) u(2 u+n v)} \\
\frac{\partial f_{k}}{\partial v} & =\frac{k(n(n+3) v-2(n-1) u) f(u, v)}{(n+1) \cdot v(2 u+n v)}
\end{aligned}
$$

Solving this system we get that

$$
f_{k}=c_{k} u^{m} v^{\frac{k-k n}{n+1}}(2 u+n v)^{2 k},
$$

with $c_{k} \in \mathbb{R}, k(1-n) /(1+n) \geq 0$ and $m \geq 0$. So it is impossible that $f_{k}$ be a homogeneous polynomial of degree $k$. This completes the proof of the lemma.

Proof of the "only if" part of Theorem 1. By Lemma 11 system (2) with $n \notin\{-6,-5$, $-3,-1\}$ has no exponential factors, and from Lemmas 5 to 10 the unique invariant algebraic curves of a such system are $u=0$ and $v=0$ with cofactors

$$
K_{1}=\frac{1-n}{2 n} u+\frac{n+3}{4} v+\frac{n+7}{2} \text { and } \quad K_{2}=-u+\frac{1}{2} v+1
$$

respectively. So, from Theorem 4 if a such system is Liouvillian integrable we must have either

$$
\lambda_{1} K_{1}+\lambda_{2} K_{2}=0
$$

or

$$
\lambda_{1} K_{1}+\lambda_{2} K_{2}=-\operatorname{div}(P, Q)
$$

with $\lambda_{1}^{2}+\lambda_{2}^{2} \neq 0$, and being $P$ and $Q$ the polynomials which appear in (2). But any of these two last equations have a solution when $n \notin\{-6,-5,-3,-1\}$. This completes the proof. 


\section{ACKNowledGements}

The second author is partially supported by the MINECO/FEDER grant MTM200803437, AGAUR grant 2009SGR-410, ICREA Academia, FP7-PEOPLE-2012-IRSES 316338 and 318999, and a grant FEDER/UNAB10-4E-378.

\section{REFERENCES}

[1] M.S.R. Delgaty And K. LAKe, Physical acceptability of isolated, static, spherically symmetric, perfect fluid soluions of Einstein's equations, Comput. Phys. Commun. 115 (1998), $395-415$.

[2] R.C. Tolman, Static solutions of Einstein's field equations for spheres of fluid, Phys. Rev. 55 (1939), 364-373.

[3] B.V. Ivanov, Integrable cases of gravitating static isothermal fluid spheres, J. Math. Physics 43 (2002), 1029-1043.

[4] H.A. Buchdahl, Reciprocal static solutions of the equations of the gravitational field, Aust. J. Phys. 9 (1956), 13-18.

[5] M.F. Singer, Liouvillian first integrals of differential equations, Trans. Amer. Math. Soc. 333 (1992), 673-688.

[6] F. Dumortier, J. Llibre And J.C. Artés, Qualitative theory of planar differential systems, UniversiText, Springer-Verlag, New York, 2006

[7] C. Christopher, J. Llibre and J.V. Pereira, Multiplicity of invariant algebraic curves and Darboux integrability, Pacific J. Math. 229 (2007), 63-117.

1 EnEA-C. R. Casaccia, Via Angulllarese 301, 00123 Roma, Italy

E-mail address: roberto.iacono@enea.it

2 Departament de Matemàtiques, Universitat Autònoma de Barcelona, 08193 Bellaterra, Barcelona, Catalonia, Spain

E-mail address: jllibre@mat.uab.cat 